\title{
Detailed analysis of combustion process of a single aluminum particle in air using an improved experimental approach.
}

\author{
Alexandre BRACONNIER ${ }^{1,2, *}$, Christian CHAUVEAU ${ }^{2}$, Fabien HALTER ${ }^{2}$, Stany GALLIER ${ }^{1}$ \\ ${ }^{1}$ ArianeGroup, CRB, 9 rue Lavoisier, 91710 Vert-Le-Petit, France \\ ${ }^{2}$ ICARE-CNRS, 1 C avenue de la recherche scientifique 45071 Orléans Cedex, France
}

alexandre.braconnier@cnrs-orleans.fr (A. Braconnier) - christian.chauveau@cnrs-orleans.fr (C. Chauveau) - fabien.halter@cnrs-orleans.fr (F. Halter) - stany.gallier@ariane.group (S. Gallier)

\begin{abstract}
The current experimental study aims at analyzing the self-sustained combustion of 30-120 $\mu \mathrm{m}$ Al particles using an electrostatic levitation system. In this improved set-up, a single particle is isolated in air with different ambient pressure (1-31 bar) to be then ignited by a $\mathrm{CO}_{2}$ laser beam. The integrated optical signature of burning Al particle is recorded using filtered photomultipliers and a high-speed camera. The photomultiplier is used to estimate burning time while the camera direct visualization allows to measure the evolution of combustion parameters in real time such as droplet diameter (including initial particle diameter) and flame diameter with important resolution. Important information are also provided to accurately describe combustion phenomenology. Measured burning times according to the pressure are compared to experimental correlations issued from literature and similar trends are observed. The existence of different stages during combustion is clearly evidenced and early assumptions can be made to explain dependence between combustion time and identified phenomena.
\end{abstract}

KEY WORDS: Solid propellant, Metal combustion, Aluminum, Burning time 


\section{INTRODUCTION}

Metal combustion has an interesting energetic potential and generate elevated combustion temperatures. According to its high energetic density and its wide availability, aluminum micrometric particles are currently used in industrial solid propellants, especially to increase thrust of the solid rocket motors (SRM). However, the combustion of aluminum particles in SRM is a complex process. Particles leave the propellant surface and burn in a specific gaseous environment, under high temperatures and pressures, given by solid propellant combustion. It is also well known that a significant part of initial aluminum particles coalesces into larger agglomerate (Price et al. 1979). Considering these conditions, combustion dynamics can be directly altered, both for solid propellant and aluminum particles. The study of the aluminum combustion phenomenon has therefore been a continuous research effort, even in more common atmospheres such as air.

Since work proposed by Friedman and Maček (1962) using a flame plate burner to ignite aluminum particles and collect quantitative and qualitative information on their combustion, several experimental apparatus have been developed to improve the understanding of the aluminum combustion with increasing accuracy. Shock tubes were often used and permit to analyze the combustion of dust aluminum considering interaction effects between burning particles (Servaites et al. 2001) (Bazyn et al. 2006) and (Lynch et al. 2007). Combustion of single particles was also studied in case of free-falling droplet, aerodynamic levitators or bulk aluminum samples attached to support, considering convective and conductive effects (Wilson and Williams 1971), (Prentice 1974), (Bucher et al. 1996), (Sarou-Kanian et al. 2003) and (Feng et al. 2016). Thus, it is proved that the combustion regime, and therefore the burning time, depends on the initial particle diameter $D_{0}$. Indeed, for large particles $\left(D_{0}>\right.$ $10 \mu \mathrm{m})$, aluminum burns as a vapor flame in diffusion mode whereas combustion of smaller 
particles occurs in a kinetic-limited regime (Bazyn et al. 2007). Determination of correlations between burning time and influencing parameters has been an ongoing effort. The assumption of a similar behavior between solid aluminum particles and liquid fuel droplets was established by Glassman (1959) and motivated the use of the classical $\mathrm{D}^{2}$ law but studies have shown that the phenomenology of the aluminum particle combustion is different from the symmetrical and steady behavior of liquid droplets. Prentice (1974) concluded that a nonsteady state must be considered to successfully describe metal droplet burning process and Dreizin (1996) identified three distinct stages in aluminum combustion history: spherically symmetric vapor phase, asymmetric regime and extinction. Relying on experiments, Beckstead (2004) proposed to consider the effect of the oxidizer efficiency pressure and ambient temperature on the burning time in an improved $\mathrm{D}^{\mathrm{n}}$ correlation, with $1.5<\mathrm{n}<1.8$.

More recently, Gill et al. (2010), Badiola and Dreizin (2012) and Feng et al. (2016) worked on the characterization of the aluminum combustion in various mixtures or pressures. Focused on small micron sized particles $\left(\mathrm{D}_{0}<25 \mu \mathrm{m}\right)$ or coarser particles $\left(100<\mathrm{D}_{0}<300\right.$ $\mu \mathrm{m})$ with different experimental approaches. These investigations highlight that some data on aluminum combustion stay scarce, often inconclusive. Indeed, even if similar trends can be proposed on the evolution of the burning time according to initial droplet diameter, pressure, or chemical composition of the ambient environment, determination of the exponent of the particle diameter according to conditions sometimes stay heterogeneous because of the various authors' experimental approaches and determination of a universal burning time correlation based on fundamental analysis is still required. Moreover, experimental limitations prevent to obtain details, especially in real time and fundamental study of the aluminum combustion process requires elaborated experimental conditions in order to avoid any perturbing aspect like heat transfer by convective effect (in case of free-falling set-up and 
aerodynamic levitator) or conduction (in case of aluminum sample on support). Thus, research must be extended to have a complete understanding of the aluminum combustion process.

In this work, an improved experimental set-up based on previous works by Marion et al. (1996) is proposed. It allows recording the combustion of a single levitated aluminum droplet in a static gaseous environment without any perturbing support. With a high temporal and spatial resolution, this set-up introduces a new method to evaluate the burning time and interaction mechanisms between the aluminum and its oxide. This paper addresses the combustion of levitated aluminum particles, varying pressure (1 to 31 bar) and on a wide representative range of particle size (30 to $120 \mu \mathrm{m}$ ). This study is devoted to providing new and more accurate experimental data, especially on the impact of the pressure using latest imaging technologies.

\section{EXPERIMENTAL SETUP}

\subsection{Isolation and ignition of a single aluminum particle in a controlled ambient environment}

Isolation of a single charged particle without any perturbing support is an important issue. Based on previous theory and design of Davis (1987) and Bar-Ziv and Sarofim (1991), an electrodynamic levitator was developed, consisted of using the electric force to oppose gravity. The levitator requires three distinct electrodes (top, bottom and central ring), supplied by $\mathrm{AC}$ and $\mathrm{DC}$ voltage to generate an electromagnetic field and provide the necessary stabilizing effect on the particle (Figure 1).

The central electrode supplied by AC voltage induces a centripetal effect to adjust the horizontal position of the particle. The vertical particle position is fixed by the DC voltage which compensates the gravity effect. In this configuration, this apparatus offers capabilities 
to suspend a motionless charged particle. Hence, a single burning particle can be observed easily in a static oxidizing atmosphere and combustion is not affected by any additional heat exchanges (convective or conductive).

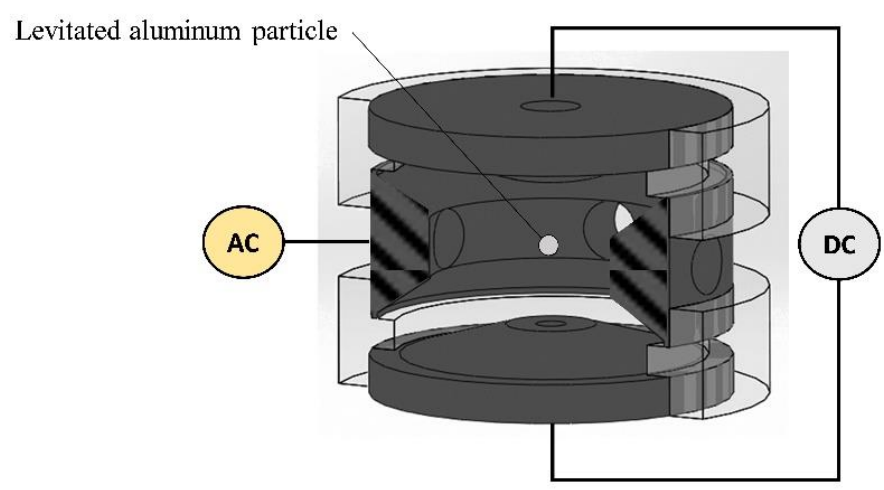

Figure 1 : Schematic illustration of electrodynamic levitator

During the combustion, the particle as well as the combustion products contained in the flame are heated to high temperatures and a thermionic emission can occur, resulting in important charge losses. Charge losses preserve the combustion process of any influence due to electric voltages, which means that the charges are unlikely to affect the combustion and are only used to ensure a fixed and reproducible initial position. Then, even if thermionic emission prevents to maintain the electromagnetic effect on burning particle to control its position, characteristic time of combustion is less than the characteristic free falling time of a heated particle and overall combustion reproducibly takes place in the observation area.

The characterization of dependence between aluminum combustion and ambient conditions in terms of temperature, pressure and composition requires controlling each parameter accurately. The technical solution is a high-pressure chamber (Figure 2) supplied with various gases from external high pressure tanks. This chamber is connected to a digital manometer permitting to control internal pressure at room temperature, ranging from 1 to 120 
bar. Molar composition of desired gaseous environment is also controlled by the manometer, using partial pressures.

A focused 50 Watt $\mathrm{CO}_{2}$ laser beam impacts the particle to initiate aluminum combustion. A beam splitter coupled to a set of mirrors is used in order to separate and reflect the laser beam and symmetrically irradiate the particle (Figure 2) while limiting its displacement. Specific $\mathrm{Zn}$-Se lenses are used to ensure a high transmission in the laser beam wavelength $(10.6 \mu \mathrm{m})$.

\subsection{Optical setup}

Main measurements used to describe the burning process are based on optical diagnostics. Indeed, a first optical setup is composed of two photomultipliers (PM) tubes (Figure 2). In more details, the luminous visible emission of the particle is collected through a lens set before to be split, filtered and integrated to finally obtain amplified optical signature of each combustion test. Typical wavelengths are selected (488 $\mathrm{nm}$ and $514 \mathrm{~nm}$ ) to mainly receive the strong radiation of $\mathrm{AlO}$ products which are characteristic of the aluminum burning phase. A high-speed camera, coupled with a long-distance microscope (Figure 2), is also focused on the single particle to observe it in real time during the combustion process, included ignition. Videography is recorded with a considerable rate up to $25000 \mathrm{fps}$ and a high resolution (1.7 to $2.5 \mu \mathrm{m} / \mathrm{px}$ ), allowing to determine a fine evolution of combustion parameters such as droplet diameter and flame diameter to estimate burning rates and times.

The experimental procedure is elaborated to record and to process optical data of an autonomous combustion. To ensure that no extra energy is provided to the burning particle, the $\mathrm{CO}_{2}$ laser is connected with the photomultipliers signal to trigger the laser beam extinction once the luminous emission from the flame is detected being beyond a fixed threshold. Moreover, photomultipliers and camera recordings are synchronized with the laser beam trigger. An in-house MatLab script has been developed to determine the significant 
parameters with an automatic data processing and image analysis. This method will be described in the next part.

\section{RESULTS}

\subsection{Measurements and data processing}

\subsubsection{Initial particle diameter}

The initial particle diameter is estimated with an image processing routine based on video sequences of aluminum combustion. Before the heating up due to the energetic laser beam,

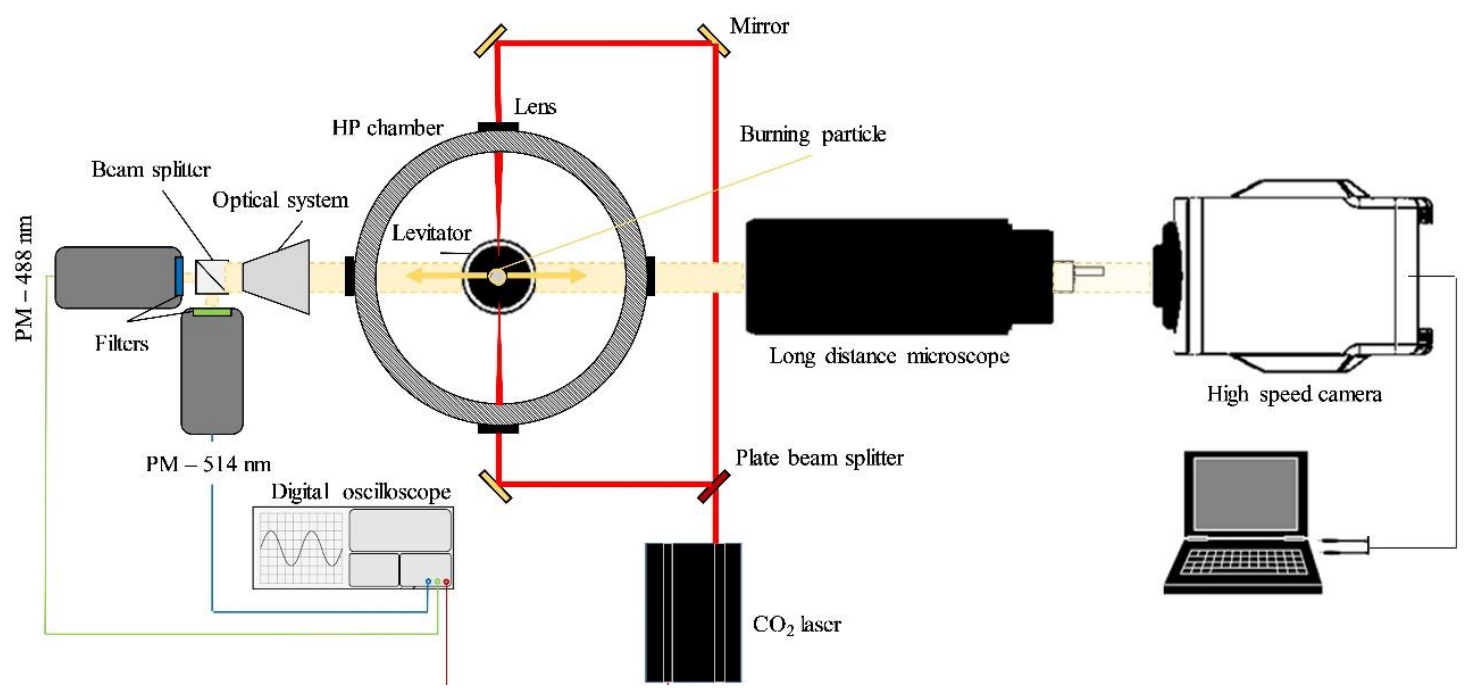

Figure 2 : Schematic representation of the experimental setup

the solid aluminum particle has an irregular shape which does not allow a reliable diameter measurement. The picture to be processed is selected when the particle forms a spherical droplet due to aluminum melting, just before the ignition (Figure 3) to avoid approximations and assumptions. Then, the determination of the initial size of the particle is based on the interpretation of light intensity profile, defining the droplet border as the most marked 
intensity gradient. Considering emission disparities on the droplet surface, due to a random distribution of brighter zones (possibly aluminum oxide), the gray level is extracted on several measured profiles crossing the droplet, typically on 35 radial profiles. The extremums of their derivatives allow defining representative detection of the droplet surface and its diameter (Figure 3). Thus, the initial diameter can be deducted, being the average of the 35 measured values.

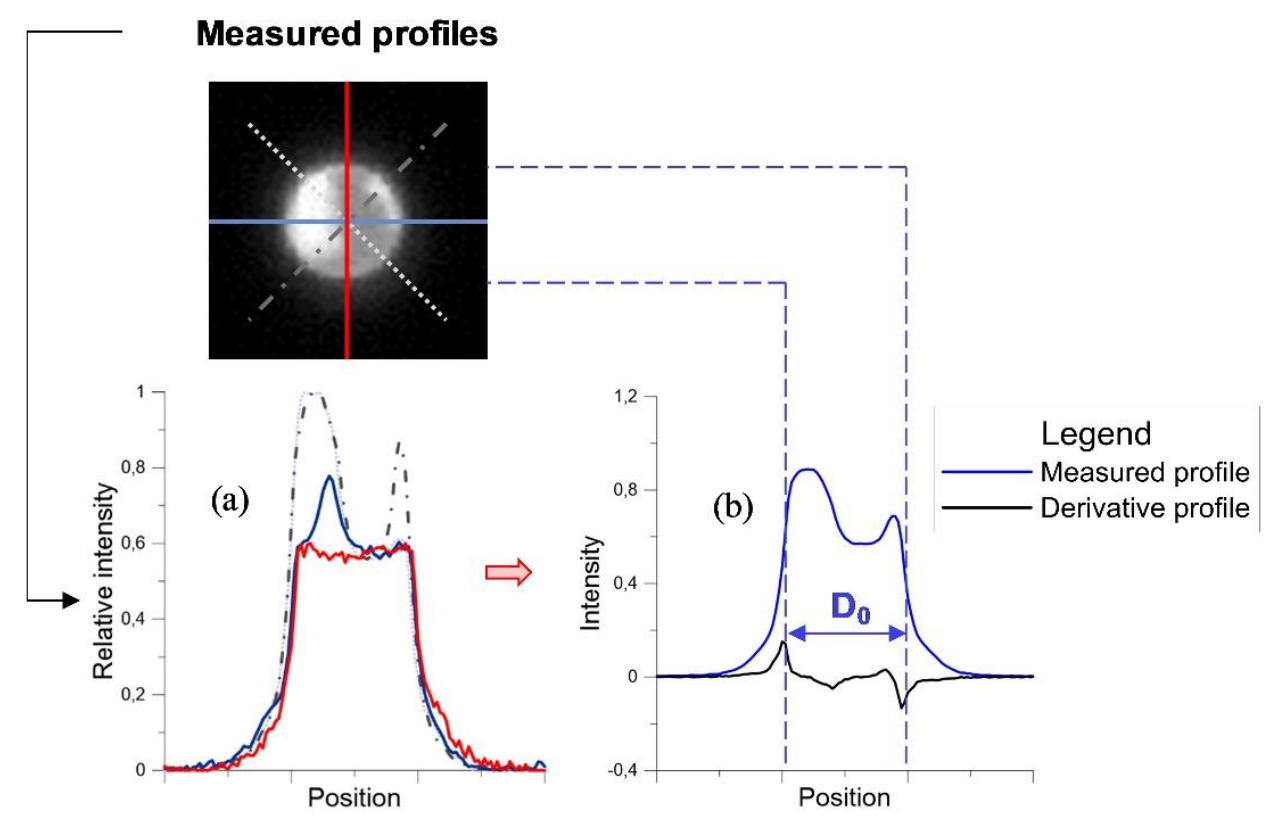

Figure 3 : Determination method of the initial droplet diameter for a burning aluminum particle in air $-\mathrm{P}=1$ bar $-\mathrm{D}_{0}=90 \mu \mathrm{m}$. Different measured intensity profiles (a). Average profile and its derivative (b).

\subsubsection{Evolution of real-time particle and flame diameter}

High-speed camera video provides an important number of images describing the combustion process with a temporal resolution less than $100 \mu \mathrm{s}$. Each picture is then processed from heating phase to extinction to determine parameters relative to the aluminum droplet combustion in real time. The methodology used is similar to that previously presented. The spherical droplet and the diffusion flame can be clearly distinguished (Figure $4-1$ ). First, a 
large zone of interest is created, based on the detection of a slightly bright circular structure around the reaction zone with a thresholding method (Figure $4-1$ ). Then, the flame area can be defined as a thermal limit. Considering this definition and according to the experimental results proposed by Bucher et al. (2000) and adapted by Glumac et al. (2005), radial temperature decreases when $\mathrm{AlO}$ consumption reduces, corresponding to the zone near the maximum $\mathrm{Al}_{2} \mathrm{O}_{3}$ mole fraction. Optically, this limit coincides with the brightest border observed in the reaction zone and this criterion will be selected to be a satisfying evaluation of the flame diameter. Thus, using Abel inversion all around the zone of interest, flame size is calculated as being the average distance between maximums of Abel inversion profiles in the reaction zone (Figure $4-$ a) excluding high-intensity singularities (like a possible aluminum oxide aggregate in the considered case) by an intermediate thresholding process (Figure 4 2).

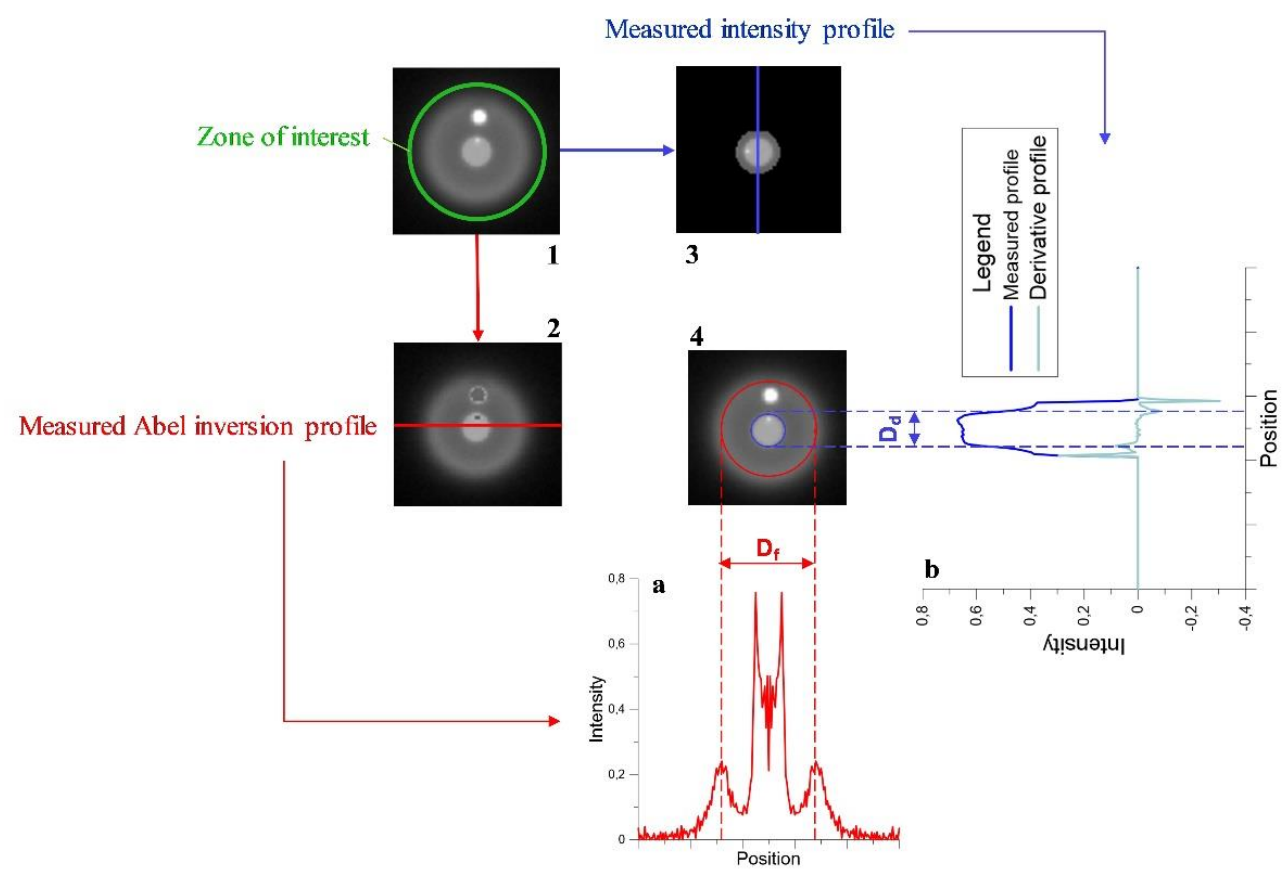

Figure 4 : Determination method of the flame and droplet diameter of a burning aluminum particle in air $-\mathrm{P}=6 \mathrm{bar}-\mathrm{D}_{0}=68 \mu \mathrm{m}$. Measured Abel inversion profile (a). Measured intensity profile and its derivative (b). 
Determination of the droplet diameter during burning phase requires additional steps. As mentioned below, flame can contain some luminous elements which can clearly affect the intensity profile and droplet detection. The solution consists in creating a mask on the flame zone, based on the previous flame diameter measurement and reported standoff ratios (Figure 4 -3). Finally, a configuration similar to the determination of the initial diameter is obtained and the same process is used. Second peaks of derivation profile are considered to represent the droplet diameter, the large first peaks being due to transition between the black mask and the surrounding bright zone (Figure $4-\mathrm{b}$ ). The results of the data processing measurements are directly superimposed on the processed picture to verify the measurement reliability (Figure $4-4)$.

\subsubsection{Burning time definition}

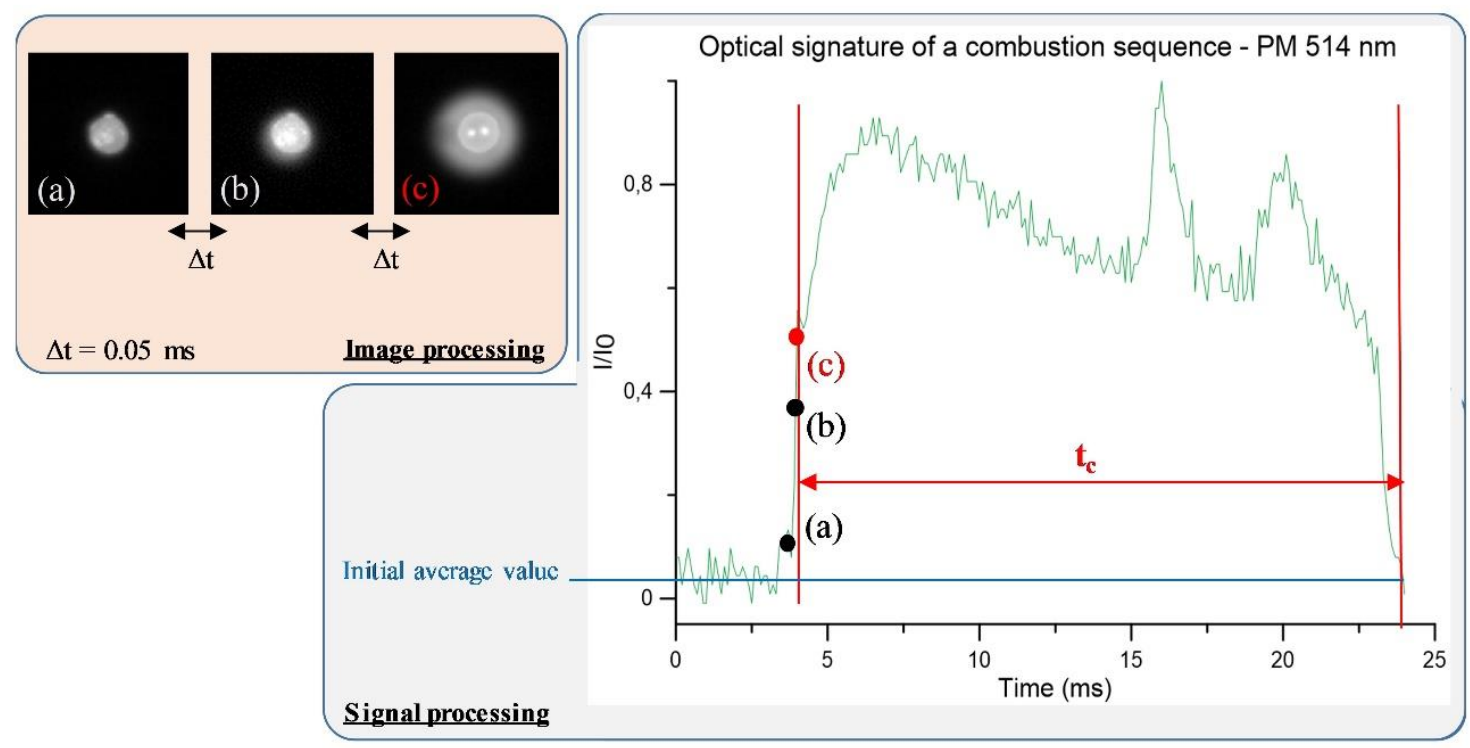

Figure 5 : Determination of combustion time based on direct visualization and emission profile recorded from a burning aluminum particle in air $-\mathrm{P}=1$ bar $-\mathrm{D}_{0}=93 \mu \mathrm{m}$. Heating phase (a) and (b). Ignition (c). 
Ignition is clearly observed with the high-speed camera, coinciding with the first picture where the diffusion flame takes place around the melted aluminum particle. Extinction corresponds to the end of the combustion process, identified when the intensity of the photomultiplier signal recovers its initial value, without any light emission. Thus, the burning time is defined using synchronized information of the high-speed camera and photomultipliers to estimate time between ignition and extinction, respectively determined with image processing and signal processing (Figure 5).

\subsection{Aluminum combustion dynamics and pressure effects}

The combustion of aluminum particles in air with varying pressure has been already investigated in different studies (Bazyn et al. 2006), (Lynch et al. 2007), (Marion et al. 1996) and (Legrand et al. 2001). The theory does not predict the effect of pressure on the combustion time in diffusion regimes but experiments identified a weak effect for low pressure range, influencing melting and vaporization temperatures and potentially combustion dynamics (Legrand et al. 2001). The interest of this study is to provide finer qualitative observations of the pressure impact on phenomenology and an accurate data set in a diameter range representative of particle diameters used in SRM.

The understanding of pressure effects in the diffusion regime requires to define clearly the combustion process of aluminum particles under atmospheric pressure in air, considered here as a well-documented reference configuration. As already reported, different combustion phases can be distinctly identified (Dreizin 1996). First, after the heating phase and the ignition, a symmetric vapor phase takes place before transiting to an asymmetric vapor phase, leading to the extinction.

The heating phase is characterized by an increasing light emission of the solid particle due to increasing temperature. Aluminum surface is naturally covered with an aluminum oxide layer (aluminum oxide $\left.\mathrm{Al}_{2} \mathrm{O}_{3}\right)$. When the melting point of aluminum is reached $(\sim 930 \mathrm{~K})$, the solid 
particle is transformed into a spherical liquid droplet due to the surface tension effect. The temperature increases, the melting point of alumina is then reached $(\sim 2300 \mathrm{~K})$ and the protective layer is finally broken and melted, staying on the aluminum droplet and forming typical distinct oxide caps on the droplet surface (Figure $6-2$ ). The gaseous aluminum can thus react with the oxidizing environment, and the combustion process starts (Figure $6-2$ ).

Then, a diffusion flame develops around the liquid droplet during the beginning of post ignition phase (Figure $6-3$ ). The flame initially grows up with the reacting gaseous flow to reach an equilibrium position and become quickly established and totally spherical, corresponding to the maximum of light emission (Figure $6-4$ ). Thus, the aluminum burns in a symmetric steady vapor phase. The droplet diameter and the flame diameter gradually reduce and the emission signal decreases proportionally to the reduction of the luminous surface (Figure $6-3$ to 6 ). However, a contour detection by the application of a Laplacian filter allows to observe the alumina lobe expansion. This image processing approximates the second derivative in both directions of the image with a convoluted $5 \times 5$ mask. The Laplacian operator is then computed and represented as a gray scale image to allow the contour detection. This method highlights that the oxide cap becomes more spread and grows up on the aluminum droplet surface, progressively creating an increasing area where the gaseous aluminum cannot be released from the droplet surface (Figure 6-5'to 6'). During the steady phase, the covered surface stay limited and does not alter the flame structure or the steady combustion process.

The transition from the symmetric steady vapor phase to the asymmetric and non-steady vapor phase is induced when the expansion of the liquid oxide on the aluminum droplet surface becomes significant (Figure $6-7^{\prime}$ ). This transition is identified by a singular intensity peak on the different emission signals. The gaseous aluminum is still produced and a gaseous flow is maintained on the reduced uncovered surface. Thus, the symmetric regime 
break induces a significant particle motion with relatively high velocity. The droplet displacement during asymmetric phase is characterized by sudden jetting (similar to thrust) and spinning effects. The spatial distribution of the reaction zone is also modified and the formation of several flame shapes occurs. A spherical flame conserved above the uncovered surface and a tapered shape above the oxide lobe (Figure $6-8^{\prime}$ ). The liquid oxide is spreading on the surface and can finally cover the whole droplet (Figure $6-9^{\prime}$ ). The synchronization between images and signals allows demonstrating that the aluminum reaction with the oxidizer is quickly prevented after this transition. In these conditions, the combustion cannot be sustained and the light emission decreases until the particle extinction.

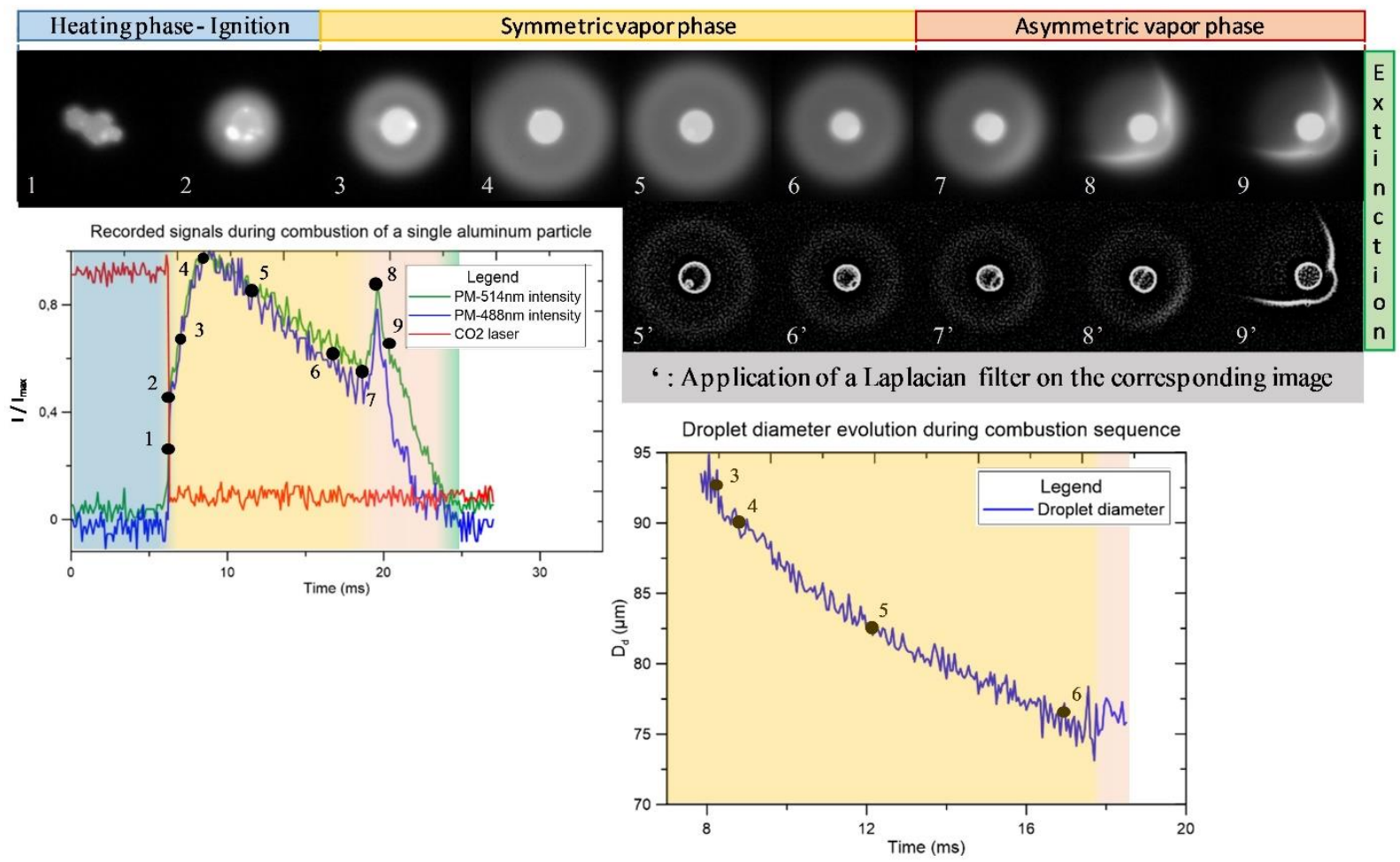

Figure 6 : Characteristic combustion sequence of an aluminum particle in air - $\mathrm{P}=1$ bar $\mathrm{D}_{0}=93 \mu \mathrm{m}$ and temporal correlation with recorded light emission profiles.

This peculiar dynamic has been only scarcely evidenced or studied and it is a strong interest of our set-up to be capable of observing it with sufficient spatial and temporal resolution. 
Works are currently ongoing to study this asymmetric combustion phase in more detail and reveal the underlying physical mechanisms. This will be the purpose of future works.

The modification of pressure does not modify the combustion steps order significantly. The existence of a symmetric vapor phase before the transition to an asymmetric regime is identified both for low and high pressures. However, the temporal distribution of the different steps and the phenomenology can be impacted. Many behaviors are observed by varying pressure (Figure 7).

Mode 1 (low pressure): the combustion process remains similar to the atmospheric case (Figure 6) but the increase in pressure contributes to an earlier asymmetric phase occurrence with a noticeable quantity of unburned aluminum. The light emission during the ignition is also more noticeable.

Mode 2 (intermediate pressure): the symmetric steady phase is significantly longer and the aluminum droplet is progressively consumed. The droplet diameter before transition to asymmetric phase is also relatively smaller due to longer symmetric combustion regime. During the symmetric regime, the accumulation of liquid oxide in the spherical flame is often observed (Figure 7). Some oxide aggregates may grow up and follow the droplet displacements until their relative size becomes important compared to the aluminum droplet or even significantly bigger. The asymmetric step finally occurs in different ways. Either, as previously mentioned, the liquid oxide cap grows up and spreads on the droplet surface or the oxide aggregate, originally located in the reaction zone, impinges the aluminum droplet, covering instantaneously a major part of its surface. In each case, jetting and spinning effects appear as well as the asymmetric flame shape before extinction. In this mode, unburned aluminum quantity is limited. 
Mode 3 (high pressure): another phenomenological aspect takes place in high pressure conditions. The flame is brighter, possibly saturated by dispersed aluminum oxide surrounding the droplet with a relative movement. Some quantities of liquid oxide can impinge the particle surface, perturbing the symmetric phase. The deposition and/or accumulation of oxide on the droplet surface generate the asymmetric transition and the particle is finally totally covered with aluminum oxide, leading to extinction in different ways. Either oxide layer is ejected like in an explosion effect before extinction or the oxide layer cannot be ejected, stays on the droplet surface and prevents the combustion further. Important quantity of unburned aluminum can be frequently observed.

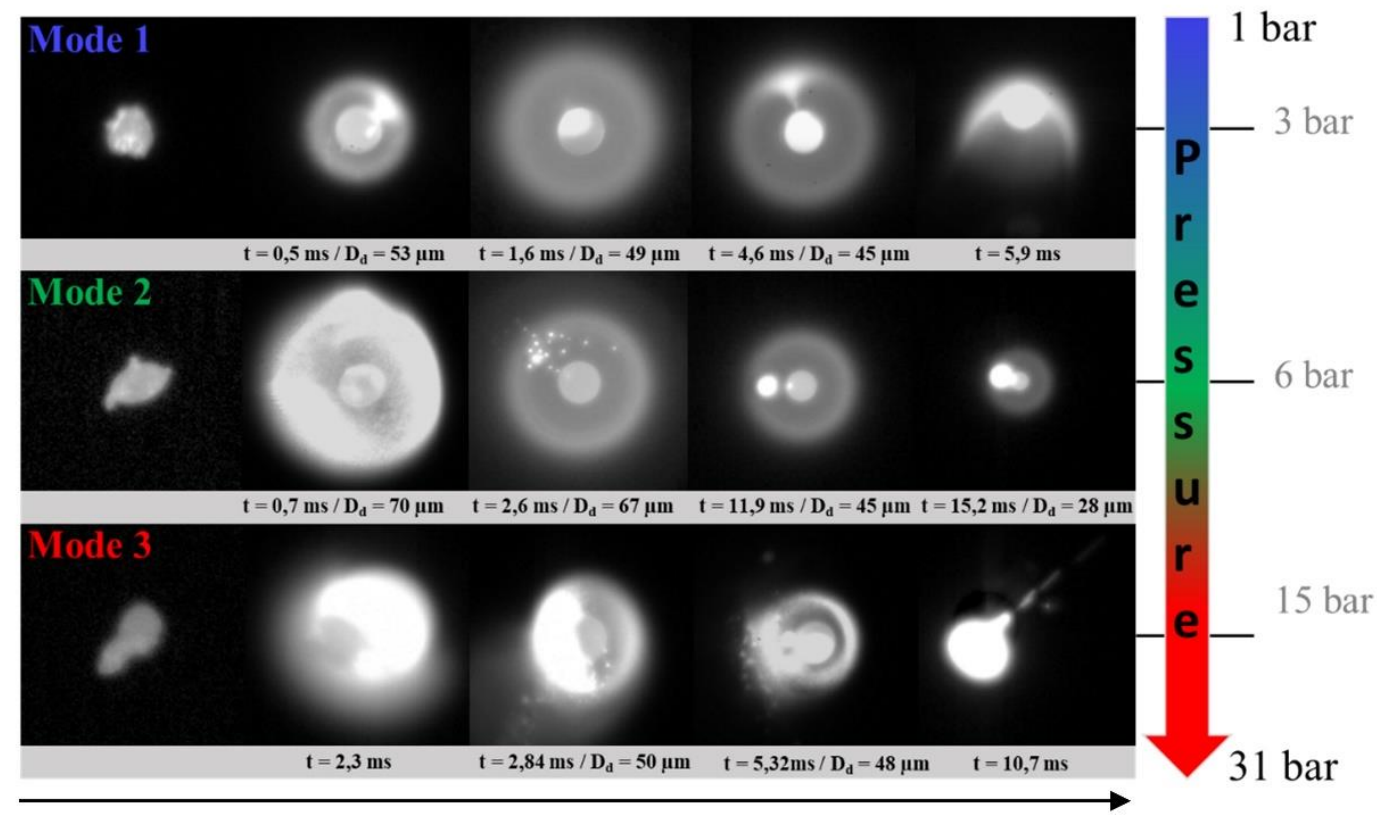

Figure 7 : Combustion sequences of aluminum particles burning in air with increasing pressure $\left(3\right.$ bar $-D_{0}=55 \mu \mathrm{m} / 6$ bar $-D_{0}=75 \mu \mathrm{m} / 15$ bar $\left.-D_{0}=53 \mu \mathrm{m}\right)$. The color intensity on the pressure scale describes the occurrence probability of each mode.

\subsection{Pressure effect on combustion parameters}


The pressure effects on combustion behavior of aluminum particles have been qualitatively described. The quantification of some parameters such as the combustion time $t_{c}$ and the relative duration of the symmetric phase allow to evaluate the pressure influence.

The determination of combustion time according to the pressure, as well as the initial droplet diameter, was established following the previously detailed methodology. A global overview of the experimental results shows that measurements are in agreement with the correlation proposed by Beckstead (2004) (Figure 8) and the observations are in accordance with documented experiments which also predict a low impact of pressure on the burning time.

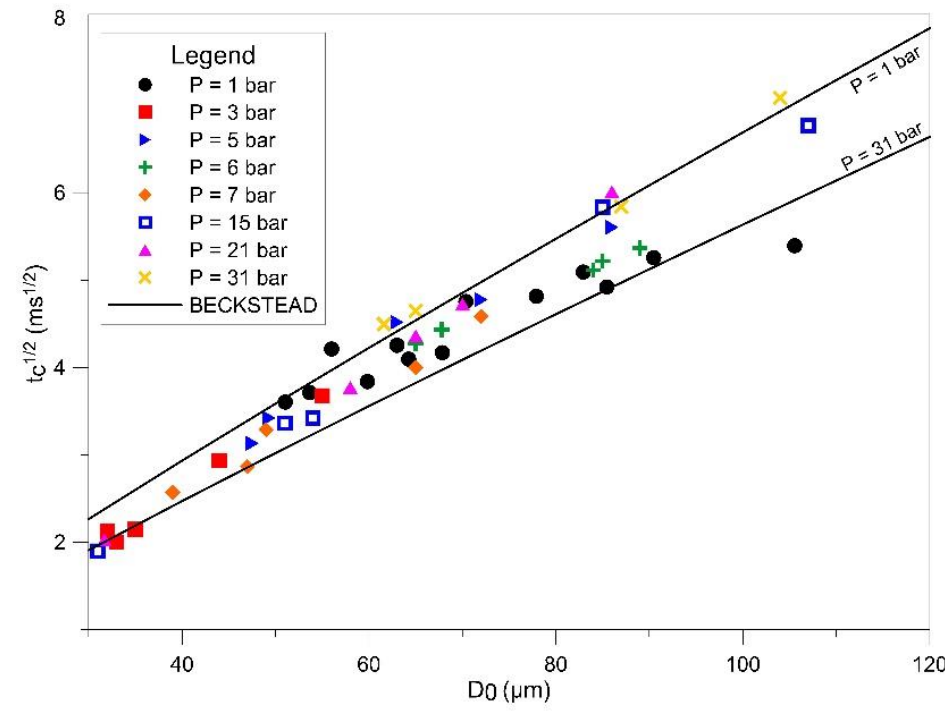

Figure 8: Aluminum combustion time dependence with pressure and initial droplet diameter. Comparison with the experimental correlation of (Beckstead 2004). Filled symbols represent experimental results and solid lines Beckstead's correlation.

A linear evolution of the combustion time according to the initial square droplet diameter is observed for each pressure range. Thus, a simplified linear correlation between $t_{c}$ and $D_{0}{ }^{2}$ is deduced for each pressure condition and used as a correction to estimate the burning time at an identical initial diameter. This measure gives another detailed trend on the burning time dependence with pressure for a given initial diameter (Figure 9). The burning time is 
relatively constant with pressure as expected from theory for a diffusion flame. However, a trend for a slight increase in combustion time with high pressures is noticed and diverges from Beckstead's results. This should be confirmed by additional measurements. Beckstead's correlation shows a decrease due to a $\mathrm{P}^{-0.1}$ dependence but his expression results from a global correlation on many experimental data and this may not capture the specificity of each case which is expected to depend on particle size, oxidizers, etc.

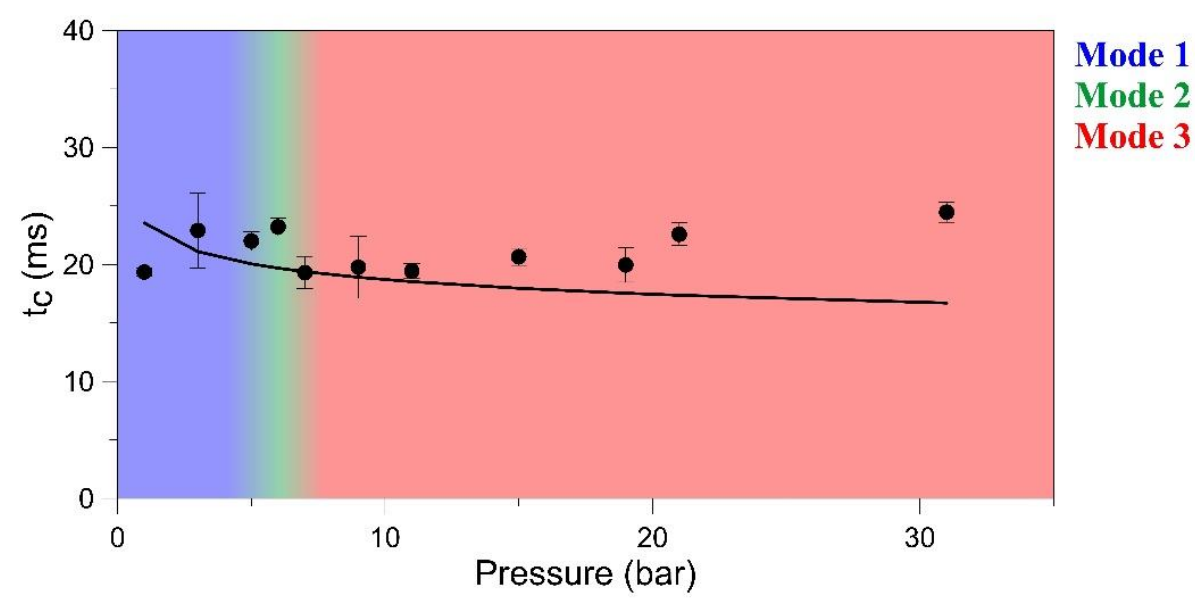

Figure 9: Aluminum combustion time dependence on pressure and comparison with experimental correlation of (Beckstead 2004). Filled symbols represent experimental results corrected by linear correlations at $\mathrm{D}_{0}=70 \mu \mathrm{m}$ and solid lines Beckstead's correlation.

The accurate information given by high-speed direct visualization allows to temporally characterize the asymmetric phase occurrence and the temporal distribution of the different burning phases is also determined to highlight the pressure impact on the phenomenology of aluminum combustion. The ignition is considered as the temporal origin and $t_{\text {avp }}$ will define the onset of the asymmetric regime and, as well, the end of symmetric combustion. The relative value of $t_{\text {avp }}$ compared to the combustion time is presented (Figure 10). These results show a potential influence of lower pressure ranges on the symmetric and steady phase duration. In first, steady phase duration reduces with pressures between 1 and 5 bar. Then a 
singular behavior is identified close to 6 bar, corresponding to the combustion mode 2 , where the duration of the symmetric phase significantly increases to represent a large part of the combustion time with a behavior similar to a liquid fuel droplet. Finally, the evolution of $\mathrm{t}_{\text {avp }}$ is then more constant and the pressure influence is predicted to be less marked for higher ranges where symmetric phase duration stays important in comparison with the combustion time. These data also corroborate the statements previously mentioned on the pressure impact on the combustion phenomenology.

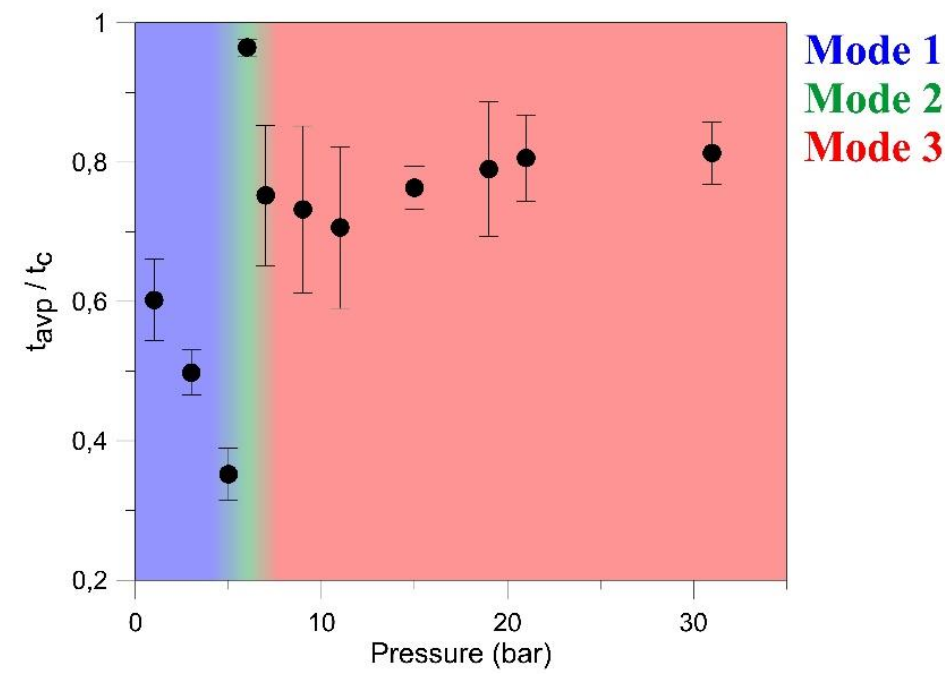

Figure 10 : Effect of pressure on temporal distribution of the symmetric and asymmetric phase related to the combustion time. Filled symbols represent an average value obtained for each pressure with several diameters. Error bars represent the standard deviation for the different diameters.

\section{DISCUSSION}

According to the experimental observations and measurements, the pressure clearly alters the combustion phenomenology, especially for lower pressures, but does not modify the burning time. Thus, if the different combustion modes are considered to interpret the evolution of $t_{c}$ according to $\mathrm{t}_{\mathrm{avp}}$ (Figure 9 and Figure 10), the assumption can be made that the combustion 
time is independent of the respective phases duration.

Various parameters can potentially explain the existence of different combustion behaviors and require further investigations. This is the objective of our ongoing efforts to understand the associated physical mechanisms in further details. In order to understand the temporal occurrence of the asymmetric phase, the accumulation process of alumina oxide on the droplet surface with respect to the pressure has to be understood and quantified. The phase transformations could be considered to have a hypothetical effect (Trunov et al. 2005) as well as the modification of the surface tensions which depend on pressure and temperature.

To explain how the combustion time can stay relatively constant despite the inconstant phase distribution the estimation of the aluminum regression rate during respective phases depending on pressure could be a first representative indicator. Indeed, pressure is predicted to have no effect on the combustion rate in a steady diffusion mode, but, investigations to confirm it and estimate this rate during the non-steady phase could bring important information. Determination of the unburned aluminum quantity is also interesting and can allow to improve the burning time definition which actually considers, in some cases, an important part of remaining aluminum fuel.

\section{CONCLUSION}

An advanced experimental set-up was developed to propose an improved approach on aluminum combustion analysis. Indeed, a quick review of the experimental research shows some limitations of the setup already used such as additional heat transfer by conduction or convection and insufficient spatial or temporal resolution. Conversely, this study provides a configuration which allows visualizing the combustion of a single aluminum particle in real time with high spatial and temporal resolution. Without any perturbing effect or support, the direct high-speed visualization of the burning particle gives considerable information on 
phenomenology. Then, the aluminum burning process can be fundamentally characterized thanks to the determination of various combustion parameters based on image processing. The results presented the unambiguous existence of symmetric and asymmetric vapor phases with accurate details. The combustion behavior is also modified due to pressure and different modes were identified. A correlation between combustion time, favorably compared with available data, and the relative duration of the symmetric phase, highlights no potential link between these parameters.

Based on this experimental set-up, further investigations are on the way to explain the existence of different combustion modes when pressure increase and quantify the effects on the combustion parameters such like standoff ratio, droplet surface regression and oxide cap expansion. 


\section{REFERENCES}

Badiola, C. and Dreizin, E. L., (2012) On Weak Effect of Particle Size on Its Burn Time for Micron-Sized Aluminum Powders, Combust. Sci. Technol., 184(12), pp. 1993-2007.

Bar-Ziv, E. and Sarofim, A. F., (1991) The electrodynamic chamber: A tool for studying high temperature kinetics involving liquid and solid particles, Prog. Energy Combust. Sci., 17(1), pp. 1-65.

Bazyn, T., Krier, H., Glumac, N., (2006) Combustion of nanoaluminum at elevated pressure and temperature behind reflected shock waves, Combust. Flame, 145(4), pp. 703-713.

Bazyn, T., Krier, H., Glumac, N., (2007) Evidence for the transition from the diffusion-limit in aluminum particle combustion, Proceedings of the Combustion Institute, 31(2), pp. 2021-2028.

Beckstead, M., (2004) A summary of aluminum combustion, Brigham Young Univ Provo Ut, http://www.dtic.mil/docs/citations/ADA425147.

Bucher, P., Ernst, L., Dryer, F., Yetter, R., Parr, T., Hanson, D. J. S. p. c., combustion, ballistics, m. i., (2000) Detailed studies on the flame structure of aluminum particle combustion, 185, pp. 689-722.

Bucher, P., Yetter, R. A., Dryer, F. L., Parr, T. P., Hanson-Parr, D. M., Viceni, E. P., (1996) Flames structure measurement of single, isolated aluminum particles burning in air, Symposium (International) on Combustion, 26(2), pp. 1899-1908.

Davis, E., (1987) Single aerocolloidal particle instrumentation and measurement, Surface and colloid science, 14, pp. 1-81.

Dreizin, E. L., (1996) Experimental study of stages in aluminium particle combustion in air, Combust. Flame, 105(4), pp. 541-556.

Feng, Y., Xia, Z., Huang, L., Yan, X., (2016) Experimental investigation on the combustion characteristics of aluminum in air, Acta Astronautica, 129(Supplement C), pp. 1-7.

Friedman, R. and Maček, A., (1962) Ignition and combustion of aluminium particles in hot ambient gases, Combust. Flame, 6, pp. 9-19.

Gill, R. J., Badiola, C., Dreizin, E. L., (2010) Combustion times and emission profiles of micron-sized aluminum particles burning in different environments, Combust. Flame, 157(11), pp. 2015-2023.

Glassman, I., (1959) Metal combustion processes, PRINCETON UNIV NJ JAMES FORRESTAL RESEARCH CENTER, Princeton, New Jersey, http://www.dtic.mil/dtic/tr/fulltext/u2/228566.pdf.

Glumac, N., Krier, H., Bazyn, T., Eyer, R., (2005) Temperature measurements of aluminum particles burning in carbon dioxide, Combust. Sci. Technol., 177(3), pp. 485-511.

Legrand, B., Marion, M., Chauveau, C., Gokalp, I., Shafirovich, E., (2001) Ignition and Combustion of Levitated Magnesium and Aluminum Particles in Carbon Dioxide, Combust. Sci. Technol., 165(1), pp. 151-174.

Lynch, P., Glumac, N., Krier, H., (2007) Combustion of 5- $\mu$ m aluminum particles in high temperature, high pressure, water vapor environments, 43rd AIAA Joint Propulsion Conference \& Exhibit, Cincinnati, OH, AIAA-2007-5643.

Marion, M., Chauveau, C., GöKalp, I., (1996) Studies on the Ignition and Burning of Levitated Aluminum Particles, Combust. Sci. Technol., 115(4-6), pp. 369-390.

Prentice, J. L., (1974) Aluminum droplet combustion: rates and mechanisms in wet and dry oxidizers, Naval weapons center china lake CA, http://www.dtic.mil/docs/citations/AD0778037.

Price, E., Mayer, W., Strahle, W., Samant, S., Powell, E., (1979) The fire environment of a solid rocket propellant burning in air, GEORGIA INST OF TECH ATLANTA, http://www.dtic.mil/docs/citations/ADA076941. 
Sarou-Kanian, V., Millot, F., Rifflet, J. C., (2003) Surface Tension and Density of OxygenFree Liquid Aluminum at High Temperature, Int. J. Thermophys., 24(1), pp. 277-286.

Servaites, J., Krier, H., Melcher, J. C., Burton, R. L., (2001) Ignition and combustion of aluminum particles in shocked $\mathrm{H} 2 \mathrm{O} / \mathrm{O} 2 / \mathrm{Ar}$ and $\mathrm{CO} 2 / \mathrm{O} 2 / \mathrm{Ar}$ mixtures, Combust. Flame, 125(1), pp. 1040-1054.

Trunov, M. A., Schoenitz, M., Zhu, X., Dreizin, E. L., (2005) Effect of polymorphic phase transformations in $\mathrm{Al} 2 \mathrm{O} 3$ film on oxidation kinetics of aluminum powders, Combust. Flame, 140(4), pp. 310-318.

Wilson, R. P. and Williams, F. A., (1971) Experimental study of the combustion of single aluminum particles in O2/Ar, Symposium (International) on Combustion, 13(1), pp. 833-845.

\section{FIGURES}

Figure $1:$ Schematic illustration of electrodynamic levitator.............................................5

Figure $2:$ Schematic representation of the experimental setup .......................................

Figure 3 : Determination method of the initial droplet diameter for a burning aluminum particle in air $-\mathrm{P}=1$ bar $-\mathrm{D}_{0}=90 \mu \mathrm{m}$. Different measured intensity profiles (a). Average profile and its derivative (b)

Figure 4 : Determination method of the flame and droplet diameter of a burning aluminum particle in air $-\mathrm{P}=6 \mathrm{bar}-\mathrm{D}_{0}=68 \mu \mathrm{m}$. Measured Abel inversion profile (a). Measured intensity profile and its derivative (b)

Figure 5 : Determination of combustion time based on direct visualization and emission profile recorded from a burning aluminum particle in air $-\mathrm{P}=1$ bar $-\mathrm{D}_{0}=93 \mu \mathrm{m}$. Heating phase (a) and (b). Ignition (c).

Figure 6 : Characteristic combustion sequence of an aluminum particle in air $-\mathrm{P}=1$ bar $-\mathrm{D}_{0}$ $=93 \mu \mathrm{m}$ and temporal correlation with recorded light emission profiles.

Figure 7 : Combustion sequences of aluminum particles burning in air with increasing pressure $\left(3 \mathrm{bar}-\mathrm{D}_{0}=55 \mu \mathrm{m} / 6\right.$ bar $-\mathrm{D}_{0}=75 \mu \mathrm{m} / 15$ bar $\left.-\mathrm{D}_{0}=53 \mu \mathrm{m}\right)$. The color intensity on the pressure scale describes the occurrence probability of each mode. .15 
Figure 8 : Aluminum combustion time dependence with pressure and initial droplet diameter. Comparison with the experimental correlation of (Beckstead 2004). Filled symbols represent experimental results and solid lines Beckstead's correlation. 16 Figure 9: Aluminum combustion time dependence on pressure and comparison with experimental correlation of (Beckstead 2004). Filled symbols represent experimental results corrected by linear correlations at $\mathrm{D}_{0}=70 \mu \mathrm{m}$ and solid lines Beckstead's correlation .17 Figure 10 : Effect of pressure on temporal distribution of the symmetric and asymmetric phase related to the combustion time. Filled symbols represent an average value obtained for each pressure with several diameters. Error bars represent the standard deviation for the different diameters. 